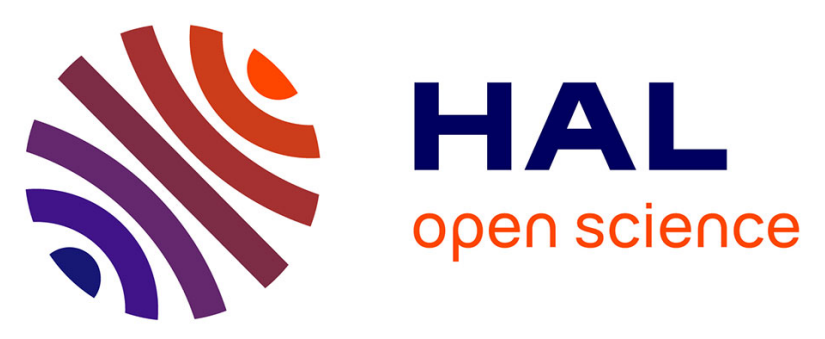

\title{
Structural and functional connections between the median and the ventrolateral preoptic nucleus
}

Augustin Walter, Lorijn van Der Spek, Eléonore Hardy, Alexis Pierre

Bemelmans, Nathalie Rouach, Armelle Rancillac

\section{- To cite this version:}

Augustin Walter, Lorijn van Der Spek, Eléonore Hardy, Alexis Pierre Bemelmans, Nathalie Rouach, et al.. Structural and functional connections between the median and the ventrolateral preoptic nucleus. Brain Structure and Function, 2019, 224 (9), pp.3045 - 3057. 10.1007/s00429-019-01935-4 . hal-03230205

\section{HAL Id: hal-03230205 \\ https://hal.science/hal-03230205}

Submitted on 19 May 2021

HAL is a multi-disciplinary open access archive for the deposit and dissemination of scientific research documents, whether they are published or not. The documents may come from teaching and research institutions in France or abroad, or from public or private research centers.
L'archive ouverte pluridisciplinaire HAL, est destinée au dépôt et à la diffusion de documents scientifiques de niveau recherche, publiés ou non, émanant des établissements d'enseignement et de recherche français ou étrangers, des laboratoires publics ou privés. 


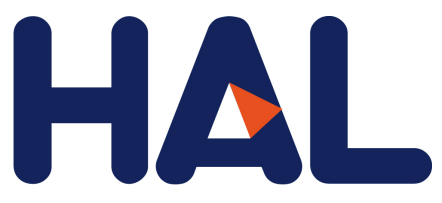

archives-ouvertes

\section{Structural and functional connections between the median and the ventrolateral preoptic nucleus}

Alexis-Pierre Bemelmans, Augustin Walter, Lorijn van der Spek, Eléonore Hardy, Alexis Pierre Bemelmans, Nathalie Rouach, Armelle Rancillac

\section{To cite this version:}

Alexis-Pierre Bemelmans, Augustin Walter, Lorijn van der Spek, Eléonore Hardy, Alexis Pierre Bemelmans, et al.. Structural and functional connections between the median and the ventrolateral preoptic nucleus: The MnPO-VLPO pathway. Brain Structure and Function, Springer Verlag, 2019, 224 (9), Epub ahead of print. 10.1007/s00429-019-01935-4 . inserm-02282038

\section{HAL Id: inserm-02282038 https://www.hal.inserm.fr/inserm-02282038}

Submitted on 9 Sep 2019

HAL is a multi-disciplinary open access archive for the deposit and dissemination of scientific research documents, whether they are published or not. The documents may come from teaching and research institutions in France or abroad, or from public or private research centers.
L'archive ouverte pluridisciplinaire $\mathbf{H A L}$, est destinée au dépôt et à la diffusion de documents scientifiques de niveau recherche, publiés ou non, émanant des établissements d'enseignement et de recherche français ou étrangers, des laboratoires publics ou privés. 


\title{
Structural and functional connections between the median
}

\section{and the ventrolateral preoptic nucleus}

\author{
Augustin Walter ${ }^{1, \#}$, Lorijn van der Spek ${ }^{1, \#}$, Eléonore Hardy ${ }^{1}$, Alexis-Pierre Bemelmans ${ }^{2}$, \\ Nathalie Rouach ${ }^{1} \&$ Armelle Rancillac ${ }^{1, *}$
}

\begin{abstract}
${ }^{1}$ Neuroglial Interactions in Cerebral Physiopathology, Center for Interdisciplinary Research in Biology, Centre National de la Recherche Scientifique UMR 7241, Institut National de la Santé et de la Recherche Médicale U1050, Collège de France, Labex Memolife, PSL-University, Paris, F-75005, France.

${ }^{2}$ Commissariat à l'Energie Atomique et aux Energies Alternatives, Département de la Recherche Fondamentale, Institut de biologie François Jacob, Molecular Imaging Research Center and Centre National de la Recherche Scientifique UMR9199, Université Paris-Sud, Neurodegenerative Diseases Laboratory, Fontenay-aux-Roses, France.
\end{abstract}

${ }^{\#}$ A. Walter and L. van der Spek contributed equally.

Abbreviated title: The MnPO-VLPO pathway.

Keywords: Sleep-promoting neuron, field recordings, VLPO, MnPO, NREM sleep, MEA

* Correspondence should be addressed to:

Dr. Armelle Rancillac

Neuroglial Interactions in Cerebral Physiopathology

Center for Interdisciplinary Research in Biology

Collège de France

CNRS UMR 7241, Inserm U1050

11 place Marcelin Berthelot 75005 Paris France

E-mail: armelle.rancillac@ college-de-france.fr 


\begin{abstract}
The median preoptic nucleus (MnPO) and the ventrolateral preoptic nucleus (VLPO) are two brain structures that contain neurons essential for promoting non-rapid eye movement (NREM) sleep. However, their connections are still largely unknown. Here, we describe for the first time a slice preparation with an oblique coronal slicing angle at $70^{\circ}$ from the horizontal in which their connectivity is preserved. Using in vivo the iDISCO method following viral infection of the MnPO or ex vivo biocytin crystal deposition in the MnPO of mouse brain slices, we revealed a strong axonal pathway from the MnPO to the VLPO. Then, to further explore the functionality of these projections, acute $70^{\circ}$ slices were placed on multielectrode arrays (MEAs) and electrical stimulations were performed near the MnPO. Recordings of the signals propagation throughout the slices revealed a preferential pathway from the MnPO to the VLPO. Finally, we performed an input-output curve of field responses evoked by stimulation of the MnPO and recorded in the VLPO. We found that field responses were inhibited by $\mathrm{GABA}_{\mathrm{A}}$ receptor antagonist, suggesting that afferent inputs from the MnPO activate VLPO neuronal networks by disinhibition.
\end{abstract}




\section{Introduction}

Compared to the large number of brain structures involved in arousal, the ventrolateral preoptic nucleus (VLPO) is considered as one of the main brain regions that turns off wake-promoting neurons during non-rapid eye movement sleep (NREM) (Economo 1930; Sherin et al. 1996; Sakai and Crochet 2001; Saper et al. 2010). Sleep neurons in the VLPO are GABAergic and galaninergic. The immunodetection of galanin forms a cluster in the VLPO allowing its localization.

Recently, another brain region composed of GABAergic neurons located in the medullary parafacial zone (PZ) was shown to be required for normal NREM sleep (Anaclet et al. 2014). Lesional experiments suggested that the VLPO could regulate NREMS sleep duration, while PZ could be involved in the regulation of the number of NREM episodes.

The median pre-optic nucleus (MnPO) is another region involved in sleep regulation. Located along the midline and dorsal compared to the VLPO, this region also contains GABAergic sleep-active neurons that are c-Fos positive following a NREM sleep hypersomnia (Gong et al. 2004). In the VLPO as in the MnPO, the number of c-Fos neurons is proportional to NREM sleep duration prior the sacrifice of the mouse.

In good agreement with this distribution of immunoreactive neurons for c-Fos, unitary recordings in the preoptic area revealed in the VLPO the existence of neurons discharging more frequently during the NREM sleep than during wakefulness (Szymusiak et al. 1998; Takahashi et al. 2009). In contrast to VLPO neurons that display an increase in discharge over an individual NREM period, MnPO sleep-active neurons discharge more frequently at the start of the NREM period (Alam et al. 2013). Therefore, the general hypothesis is that MnPO neurons play a more important role in NREM onset, whereas VLPO neurons are important for maintaining sleep. 
Indeed, retrograde transport experiments identified a set of GABAergic neurons in the VLPO, containing the peptide galanin, which innervate all of the major components of the ascending arousal systems (Sherin et al. 1998; Saper et al. 2005).

Retrograde labeling experiments previously revealed that MnPO neurons strongly project towards the VLPO (Chou et al., 2002; Thompson and Swanson, 2003, Uschakov et al., 2006; Uschakov et al., 2007). This pathway is believed to be crucial for NREM regulation, as MnPO neurons are considered to be involved in sleep pressure, whereas VLPO neurons regulate NREM onset and maintenance (Szymusiak et al., 1998; Takahashi et al., 2009; Gvilia et al., 2006), which may allow MnPO neurons to drive VLPO activity. However, the functionality of this pathway remained to be explored.

Here, we used the in vivo the iDISCO method to reconstruct the pathway between the MnPO and the VLPO and biocytin crystal deposition into the $\mathrm{MnPO}$ of on coronal or $70^{\circ}$ slices to validate the preparation. We show functional connectivity between the MnPO and the VLPO with MEA and field recordings. 


\section{Materials and Methods}

Animals. Male C57BL/6J mice (Janvier, France) and FVB Gal-GFP mice (Mutant Mouse Regional Resource Center (MMRRC), USA), 30 days old, were used. The Gal-GFP mouse strain STOCK Tg (Gal-EGFP) HX109Gsat/Mmucd (identification number 016342-UCD) was obtained from the MMRRC, a NIH-funded strain repository, and was donated by N. Heintz (Gong et al. 2003) to the MMRRC via the NINDS-funded GENSAT BAC transgenic project. All animals were bred and housed in a temperature-controlled $\left(20-22^{\circ} \mathrm{C}\right)$ room under a 12/12 light/dark cycle (7 am/7 pm) with ad libitum access to food and water. Animals used for ex vivo studies were placed in light-tight ventilated cabinets and sacrificed at ZT-0. All animal procedures were conducted in strict compliance with our institutional protocols and were approved by the European Community Council Directive of 1 January 2013 (2010/63/EU) and the local ethics committee (C2EA-59, 'Paris Centre et Sud') and local guidelines for the ethical treatment of animal care (Center for Interdisciplinary Research in Biology in College de France, France). The number of animals used in our study was kept to the necessary minimum.

$\boldsymbol{A} \boldsymbol{A} \boldsymbol{V}$ production and injection. One GFP cassette was placed under the control of a hSynapsinspecific promoter in an AAV shuttle plasmid containing the inverted terminal repeats (ITR) of AAV2. Pseudotype serotype 9 AAV particles were produced by transient cotransfection of HEK-293T cells. Viral titers were determined by quantitative PCR amplification of the ITR on DNase-resistant particles and expressed as vector genomes per $\mathrm{mL}(\mathrm{vg} / \mathrm{mL})$.

One-month old C57B16 mice were anesthetized with a mixture of ketamine (95 mg/kg; Merial) and xylazine $(10 \mathrm{mg} / \mathrm{kg}$; Bayer) in $0.9 \% \mathrm{NaCl}$ and placed on a stereotaxic frame under body temperature monitoring. AAVs were diluted in PBS at a concentration of AAV-hSynapsin-GFP $1.02 \times 10^{13} \mathrm{vg} / \mathrm{ml}$, and $0.5 \mu \mathrm{l}$ of virus was stereotaxically injected unilaterally into the MnPO at 
a rate of $0.2 \mu 1 / \mathrm{min}$, using a 29 -gauge blunt-tip needle linked to a 2 - $\mu$ l Hamilton syringe (Phymep). The stereotaxic coordinates to Bregma were antero-posterior: $-0.3 \mathrm{~mm}$; lateral: 0 $\mathrm{mm}$; and dorso-ventral: $4.5 \mathrm{~mm}$. At the end of the injection, the needle was left in place for 5 min before being slowly removed. The skin was sutured, and mice recovery was checked for the next $24 \mathrm{~h}$. After 10 days, the mice were sacrificed, and the brains were extracted after $4 \%$ paraformaldehyde (PFA)/PBS intracardiac perfusion.

\section{IDISCO and Computer-aided reconstruction of the MnPO-VLPO pathway}

To reconstruct the MnPO-VLPO pathway, the iDISCO method was performed in mice injected with the AAV-hSYN-EGFP in the MnPO (Renier et al. 2014). Briefly, the axonal track was imaged performed using a lightsheet microscopy (Ultra Microscope II; Lavision-BioTec). Reconstructions of the staining was made using Fiji (Simple Neurite Tracer) to create a threedimensional model of this pathway. From this model, we used Fiji software to design the optimal slice preparation that could contain a functional track from the MnPO to the VLPO.

Preparation of acute hypothalamic slices. Acute slices $(250$ or $400 \mu \mathrm{m})$ were prepared as previously described (Scharbarg et al. 2016). Animals were killed by decapitation at the beginning of the light phase. Brains were quickly extracted without removing the meninges and submerged in ice-cold oxygenated $\left(95 \% \mathrm{O}_{2}-5 \% \mathrm{CO}_{2}\right)$ artificial cerebrospinal fluid (aCSF) containing (in mM): $130 \mathrm{NaCl} ; 5 \mathrm{KCl} ; 2.4 \mathrm{CaCl}_{2} ; 20 \mathrm{NaHCO}_{3} ; 1.25 \mathrm{KH}_{2} \mathrm{PO}_{4} ; 1.3 \mathrm{MgSO}_{4}$ and 10 D-glucose; 15 sucrose $(\mathrm{pH}=7.35)$. Coronal brain slices containing the VLPO were cut with a vibratome (VT2000S; Leica) whereas slices cut with an angle were glued to a wedge with a $60^{\circ}$ angle from the horizontal. The VLPO being at the bottom surface of the brain, we glued it on its dorsal face to an angled wedge. As the mouse cortex does not have a flat surface, the 
brains have been slightly inclined towards their anterior extremity. This orientation increases the cutting angle by about $10 \%$. Thus, to make $70^{\circ}$ cuts, we used an angled wedge of $60^{\circ}$.

Then, slices were transferred to a constantly oxygenated $\left(95 \% \mathrm{O}_{2}-5 \% \mathrm{CO}_{2}\right)$ holding chamber containing aCSF. Subsequently, individual slices were placed in a submerged recording chamber maintained at $32^{\circ} \mathrm{C}$ and perfused with oxygenated aCSF, and placed under an upright microscope (Zeiss) equipped with a CCD camera (Hamamatsu) for observation.

Biocytin staining, immunohistology and confocal microscopy. To validate the optimal slicing angle that preserved the connection between the MnPO and the VLPO, we performed freefloating $250 \mu \mathrm{m}$ slices on Gal-GFP mice. Brains were sliced using a $70^{\circ}$ angle, or a coronal section. Slices containing the VLPO were selected and kept at room temperature in a constantly oxygenated $\left(95 \% \mathrm{O}_{2}-5 \% \mathrm{CO}_{2}\right)$ holding chamber containing aCSF. Small biocytin crystal depositions were performed under a binocular microscope into the MnPO and then, slices were returned into their holding chamber during $1 \mathrm{~h}$. Slices were then fixed by immersion in $4 \%$ PFA (overnight; $4{ }^{\circ} \mathrm{C}$ in $0.1 \mathrm{M}$ phosphate buffer) and rinsed in PBS. For immunofluorescent detection of biocytin crystals, sections were incubated with a Strepta-555-conjugated (1:200, Invitrogen) in PBS/Gelatine/Triton $1 \%$ during 2 hours at room temperature. Sections were rinsed in PBS, mounted in Fluoromount G (Thermo Scientific). Confocal images were acquired with a spinning W1 confocal microscope (Zeiss). Each image is a z-stack max intensity projection of 5 serial images (10 $\mu \mathrm{m}$ step). The length of labeled axonal fibers detected in the VLPO was measured in both hemispheres of 3 brains cut with a $70^{\circ}$ angle and 3 others cut coronally. Quantification were assessed on $210.94 * 210.94 \mu \mathrm{m}$ ROI in both conditions. A length of at least $10 \mu \mathrm{m}$ was considered an axonal segment. 
To determine the density of glutamatergic and GABAergic synapses performed by MnPO fibers into the VLPO, we performed stereotaxic injections of biocytin $(0.2 \% ; 0.5 \mu \mathrm{L})$ into the MnPO (AP: $-0.3 \mathrm{~mm}$; ML: $0 \mathrm{~mm}$; DV: $4.5 \mathrm{~mm}$ ) of 3 Gal-GFP mice of P30, anesthetized with ketamine kylaxine (100 mg/kg and $10 \mathrm{mg} / \mathrm{kg}$, respectively). 3 days later, these mice were perfused using a 2\%PFA solution and brains were postfixed in a $2 \%$ PFA solution overnight. Brains were cut using a $70^{\circ}$ angle and slices $(200 \mu \mathrm{m}$ thick). Slices were rinsed in a PBS solution. For immunodetection of GFP, biocytin, glutamatergic and GABAergic synapses, sections were sequentially incubated with the following: (1) Guinea pig anti-VGLUT2 (1:500, Synaptic Systems), rabbit anti-Homer1 (1:500, Synaptic Systems) and rabbit anti-VGAT (Millipore), mouse anti-Gephyrin (Synaptic Systems) in PBS 1X containing gelatin (2 g/L) and Triton X$100(0.25 \%)$ overnight at $4{ }^{\circ} \mathrm{C}$; (2) Strepta-555-conjugated (1:200, Invitrogen), goat antiGuinea pig Alexa Fluor 633 (1:1000, Molecular Probes), goat anti-rabbit Alexa Fluor 405 (1:1000, Life Technologies), donkey anti-rabbit Alexa Fluor 647 (1:1000, Life Technologies), goat anti-mouse Alexa Fluor 405 (1:1000, Life Technologies) during 2 hours at room temperature. Two $30 \mathrm{~min}$ PBS/gelatin/triton $(0.25 \%)$ washes were performed between each incubation step. Finally, sections were rinsed in PBS 1X and mounted in Fluoromount G (Thermo Scientific). Confocal images were acquired with a Spinning W1 confocal microscope (Zeiss). Each image is a z-stack max intensity projection of 5 serial images (10 $\mu \mathrm{m}$ step). Analyses were made with ImageJ plugin SynapseCounter (by Andrey Rozenberg and Egor Dzyubenko). Pre and postsynapctic proteins clusters where automatically determined by the plugin and only elements corresponding to 33-100\% overlap between pre and post synaptic clusters were considered as synapses.

Field recordings. 400- $\mu \mathrm{m}$ thick-slices were maintained immersed and continuously perfused at 1-2 $\mathrm{mL} / \mathrm{min}$ with oxygenated kynurenic acid-free aCSF. Local field potential (LFP) recordings 
were performed with $0.5-1 \mathrm{M} \Omega$ glass pipettes filled with extracellular solution. Evoked responses were induced by single stimulation of afferences with incremental intensities $(10 \mu \mathrm{A}$ steps starting from 0). Field potential recordings were acquired with a Axopatch-1D amplifier (Molecular Devices), digitized at $10 \mathrm{kHz}$, filtered at $2 \mathrm{kHz}$ and analyzed with pCLAMP software (Molecular Devices).

Multi electrode array (MEA) recordings. For MEA recordings, VLPO slices of $400 \mu \mathrm{m}$ were transferred on planar MEA petri dishes (120 electrodes at $100 \mu \mathrm{m}$ interelectrode distance; Multichannel Systems). Slices were maintained in place by using a small platinum anchor and were continuously perfused $(2 \mathrm{ml} / \mathrm{min})$ with aCSF. Pictures of hypothalamical slices on MEAs were used to identify the location of the electrodes in the different brain regions and to select the electrodes of interest near the MnPO. Data were sampled at $10 \mathrm{kHz}$ and evoked network activity was recorded at $34{ }^{\circ} \mathrm{C}$ by a MEA2100-60 system (bandwidth, 1 to $3000 \mathrm{~Hz}$; gain, 2x; Multichannel Systems) through the MC_Rack 4.4.1 software (Multichannel Systems). Responses were evoked by single stimulation of afferences (15 $\mu$ A step). Stimulation artefacts were truncated in the illustration for clarity. Raw data were analyzed with MC_Rack (Multi Channel Systems) and Clampfit software (Molecular Devices).

Statistics. All data are expressed as the mean \pm standard error of the mean (SEM). Statistical analysis were performed using Statistica software (Statsoft) and GraphPad Prism (GraphPad Software, Inc). 


\section{RESULTS}

\section{Computer-aided design reconstruction of the MnPO-VLPO pathway}

Here, we first performed a reconstruction of the pathway from the MnPO to the VLPO. We injected an AAV-hSYN-EGFP into the MnPO to label MnPO neurons projecting toward the VLPO. Then, we used the iDISCO method to perform a whole-mount immunolabeling of the GFP and to image a large cleared brain region containing the MnPO and the VLPO, without invasive protocols such brain slicing (Fig. 1A-B). Using Simple Neurite Traceur (Fiji), we then generated serial-section reconstructions of the labeled fibers (Fig. 1C). The digitalized pathway was processed by Fiji software, which allowed us to rotate the reconstruct in three-dimensional space. Thus, we determined that the optimal slicing angle was $74.1^{\circ}$ from the horizontal and estimated the optimal slice thickness that included an intact axonal pathway from the MnPO to the VLPO, to be $250 \mu \mathrm{m}$ (Fig. 1C-D).


Figure 1. Reconstruction of the MnPO-VLPO pathway. A,

Schematic transversal surface views where MnPO fibers were imaged and reconstructed after AAV-hSYN-EGFP injection into the VLPO. B, Confocal images of the ROI shown in A (top traces) and at higher magnification (bottom traces). Scale bars: $200 \mu \mathrm{m}$. C, Reconstructions of the MnPO (red), the VLPO (green) and the MnPO fibers (yellow) using Fiji software (Simple Neurite Tracer plugin) and the Blue Brain Atlas (EPFL's Blue Brain Project). D, Schematic representation of a lateral view of the slicing angle $\left(90^{\circ}\right)$. 


\section{Anatomical Connectivity Between MnPO and VLPO}

To further investigate the structural and functional connectivity between the MnPO and the VLPO, we next generated and compared two different slice preparations containing the MnPO and the VLPO. In adult mice, the MnPO is located between 0.62 and $-0.14 \mathrm{~mm}$ along the midline (Fig. 2A) and dorsally compared to the VLPO that is located between Bregma 0.26 and -0.10 mm (Franklin and Paxinos 1997, The Mouse Brain in Stereotaxic Coordinates; Fig. 2B). Thus, we performed coronal slices (cutting the brain at $90^{\circ}$ ) at Bregma 0.20, including both extremities of these nuclei and angular slices (cutting the brain at $70^{\circ}$ ) as determined by the iDISCO method and that should preserve the axonal track integrity.

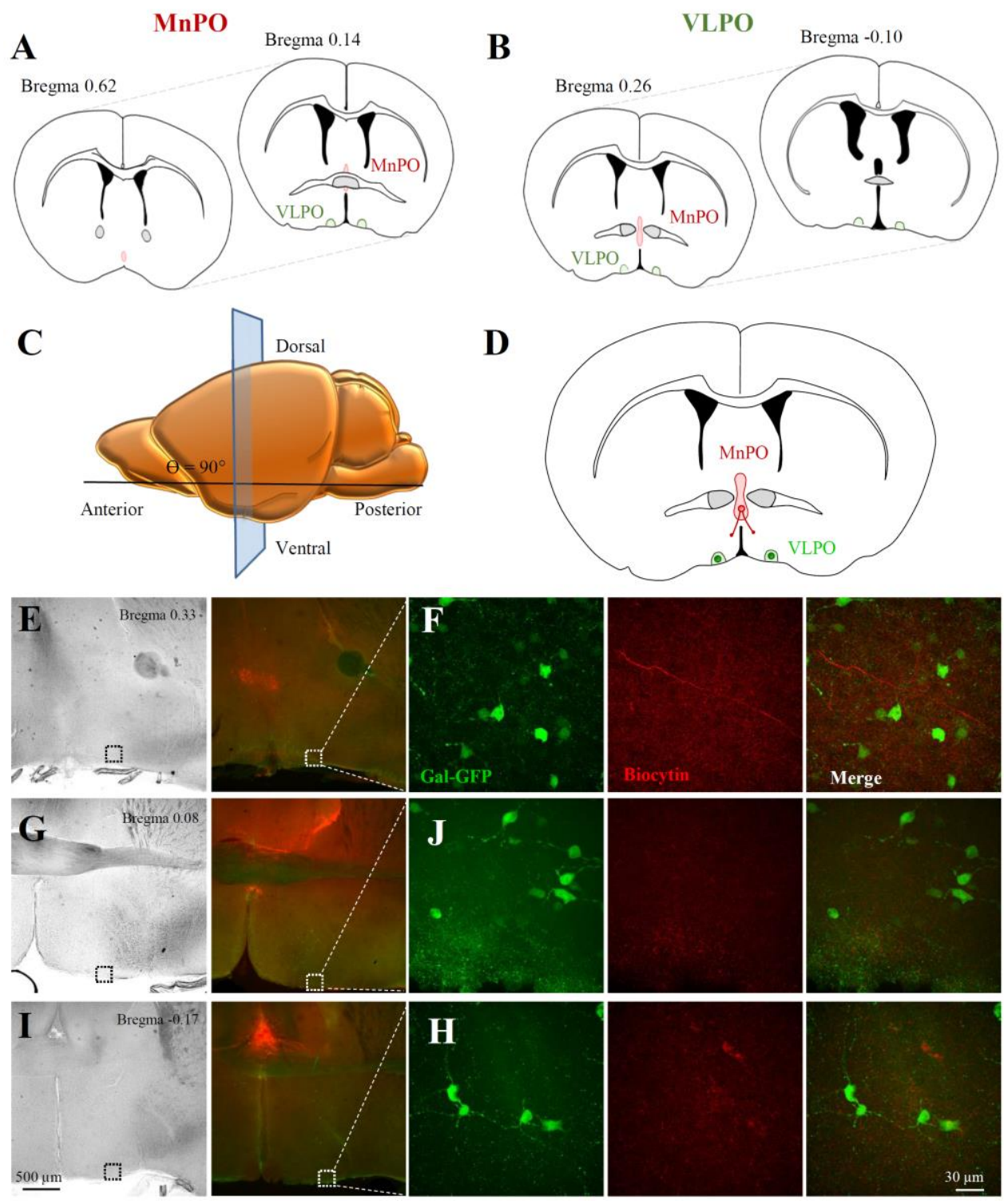


Figure 2. Biocytin-labeling of axonal projections from the MnPO to the VLPO in coronal slices. A, Schematic representation of coronal slices (Bregma 0.62 and Bregma $0.14 \mathrm{~mm}$ ) illustrating the extremity of the MnPO location (in red, from Paxinos \& Franklin, 2004). Note the VLPO shown in green. (B) Schematic representation of coronal slices (Bregma 0.26 and Bregma $-0.10 \mathrm{~mm}$ ) illustrating the extremity of the VLPO location (in green, from Paxinos \& Franklin, 2004). Note the MnPO shown in red. (C) Schematic representation of a lateral view of the slicing angle $\left(90^{\circ}\right)$. (D) Schematic representation of the corresponding slice and of the axonal projections from the MnPO (in red) to the VLPO (in green) that were cut. (E, G and I) Confocal bright field and fluorescent images of 3 slices, cut at Bregma 0.33 (E), 0.08 (G) and $0.17 \mathrm{~mm}$ (I) containing Gal-GFP VLPO neurons (green) and a biocytin labeling (red). (F, J, H) Higher magnifications of the regions indicated by a white dotted square in E, G and I. Note that biocytin labeled fibers were observed in the most rostral coronal brain section only.

To test the connectivity between the MnPO and the VLPO, we used the Gal-GFP mice that express GFP under the promoter of galanin and enables visualization of the VLPO (Gaus et al. 2002). Sleep promoting-neurons of the VLPO are indeed galaninergic. The visualization of fluorescence in galaninergic neurons thus allows an easy and robust determination of VLPO localization. First, we performed acute coronal brain slices of $250 \mu \mathrm{m}$ thickness for both orientation $\left(90\right.$ and $70^{\circ}$ ) using P30 Gal-GFP mice and next, we placed a biocytin crystal onto the MnPO of these slices that contained both the MnPO and the VLPO. This aim of these experiments was to label the fibers after the slicing, to be sure that the staining observed into the VLPO resulted only from afferent fibers contained in the thickness of the slice without any other trajectory. Thus, we placed a biocytin crystal onto the MnPO of these slices during $1 \mathrm{~h}$, to allow an anterograde transport of biocytin and the labeling of the pathway (Fig. 2).

We then examined the slices containing Gal-GFP neurons to determine whether biocytin labeled fibers from the MnPO could be observed in the VLPO. We found only few biocytinlabeled fibers and only in the slice containing the rostral part of the VLPO, at Bregma $0.25 \mathrm{~mm}$ (Fig. 2E-G). 
We then tested whether a brain slice cut using the optimal angle of $70^{\circ}$ preserved the connection between the MnPO and the VLPO. We performed another series of biocytin-crystal depositions into the MnPO of these slices, during $1 \mathrm{~h}$. Slices were then fixed and visualized using confocal microscopy. As shown in figure 3, all the slices containing Gal-GFP VLPO neurons also had biocytin labeled-fibers in the VLPO (Fig. 3C and D).

A


B
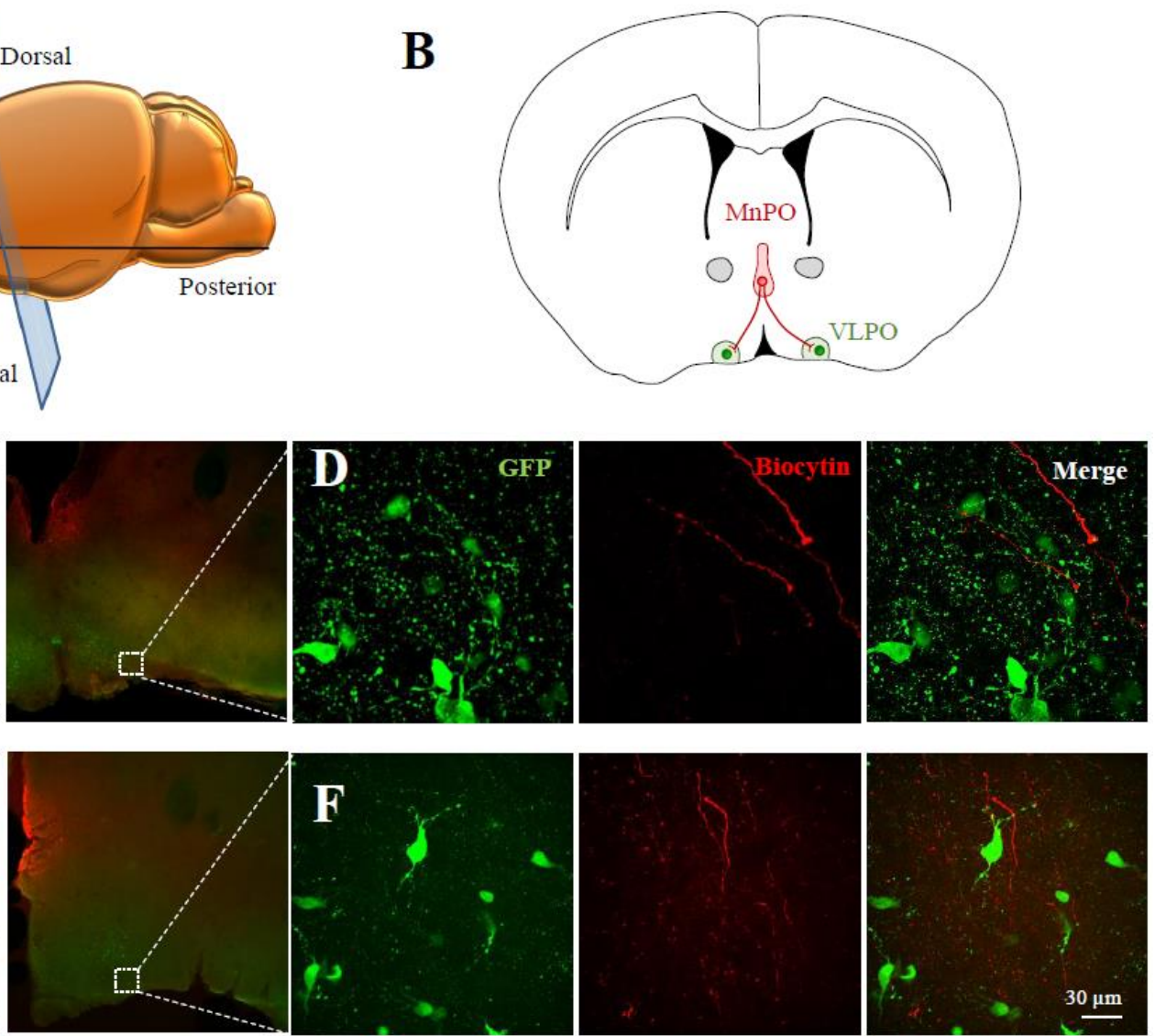

Figure 3. Biocytin-labeling of axonal projections from the MnPO to the VLPO on brain sections cut with a $70^{\circ}$ angle. A, Schematic representation of a lateral view of the slicing angle $\left(70^{\circ}\right)$. B, Schematic representation of the corresponding slice and of the axonal projections from the MnPO (in red) to the VLPO (in green) that were preserved. $\mathbf{C}$ and $\mathbf{E}$, Confocal bright field and fluorescent images of the 2 slices containing Gal-GFP VLPO neurons (green) and a biocytin labeling (red). D and F, Higher magnifications of the regions indicated by a white dotted square in $\mathrm{C}$ and $\mathrm{E}$. Note that biocytin labeled fibers were observed in all brain sections containing the VLPO. 
Comparisons of the MnPO fibers preservation in both preparations using either a slicing angle at $90^{\circ}$ (coronal slices) or at $70^{\circ}$ were performed on biocytin labeled fibers after the slicing. We observed that the MnPO fiber track was denser on $70^{\circ}$ angle brain sections than on coronal slices, suggesting a preserved integrity of the track (Fig. 4). Accordingly, we established that in the VLPO, the mean labeled length of MnPO fibers was significantly increased by $56.5 \%$ in brain slices performed at a $70^{\circ}$ angle (from $44.1 \pm 5.1$ to $72.8 \pm 4.7 \mu \mathrm{m}$; $\mathrm{P}<0.01$, MannWhitney test; Fig. 4E). In particular, the number of axonal fibers was significantly increased by $69.1 \%$ in brain slices performed at a $70^{\circ}$ angle (from $1.9 \pm 0.7$ to $10.1 \pm 2.1$; $\mathrm{P}<0.001$, MannWhitney test; Fig. 4F). Thus, we found that $70^{\circ}$ brain slices preserved the integrity of MnPO fibers arriving in the VLPO. This slicing angle allowed the preservation of more numerous and longer MnPO fibers. 



Figure 4. Biocytin labeling of the MnPO-VLPO pathway on a coronal and a $70^{\circ}$ slice. A and $\mathbf{B}$, Schematic representation of a lateral view of the coronal slicing angle at $90^{\circ},(\mathrm{A})$ and $70^{\circ}$ (B). C and D, Confocal images of non-overlapping regions of interest illustrating the biocytin labeled pathway from the MnPO (top panel) to the VLPO (bottom panel) on slices cut either at $90^{\circ}$ (C) or at $70^{\circ}$ (D). E, Individual plots and means of the length of labeled axonal fibers detected in the VLPO in slices performed at $90^{\circ}$ and at $70^{\circ}$. F, Mean number of labeled axonal fibers detected per VLPO slices in each condition. 


\section{Excitatory and inhibitory synapses are both observed in the VLPO on biocytin-labeled}

\section{fibers from the MnPO}

To investigate the putative nature of this afferent pathway to the VLPO, we performed in vivo biocytin injection into the MnPO of Gal-GFP mice. After biocytin diffusion, brains were sliced using the $70^{\circ}$ angle. Biocytin labeling was revealed and immunodetection of excitatory synapses was performed on half of the slices, using the combination of a VGLUT2 antibody to visualize presynaptic elements and a homer antibody, to visualize postsynaptic elements. In parallel, on the other slices, immunodetection of GABAergic synapses was assessed using the combination of the VGAT antibody to visualize their presynaptic elements and the gephyrin antibody, to visualize their postsynaptic elements. In the VLPO, we next determined the density of excitatory and inhibitory synapses observed on biocytin-labeled fibers from the MnPO (Fig. 5). We found that on average, axons from the MnPO formed $154.9 \%$ more excitatory synapses than inhibitory $(0.13 \pm 0.01$ vs. $0.05 \pm 0.01, \mathrm{P}<0.001$, Mann-Whitney; Fig. 5A-D). To go further into the anatomical characterization of this innervation, we quantified the synaptic densities of MnPO fibers performed on non Gal-GFP neurons and on Gal-GFP neurons. Although not statistically significant, we also observed a higher proportion of excitatory synapses on Gal-GFP neurons $\left(0.13 \pm 0.01\right.$ vs. $0.07 \pm 0.01$ synapses $/ \mu \mathrm{m}^{2}$ respectively, $P=0.061$, Kruskal-Wallis test; Fig. 5D). 


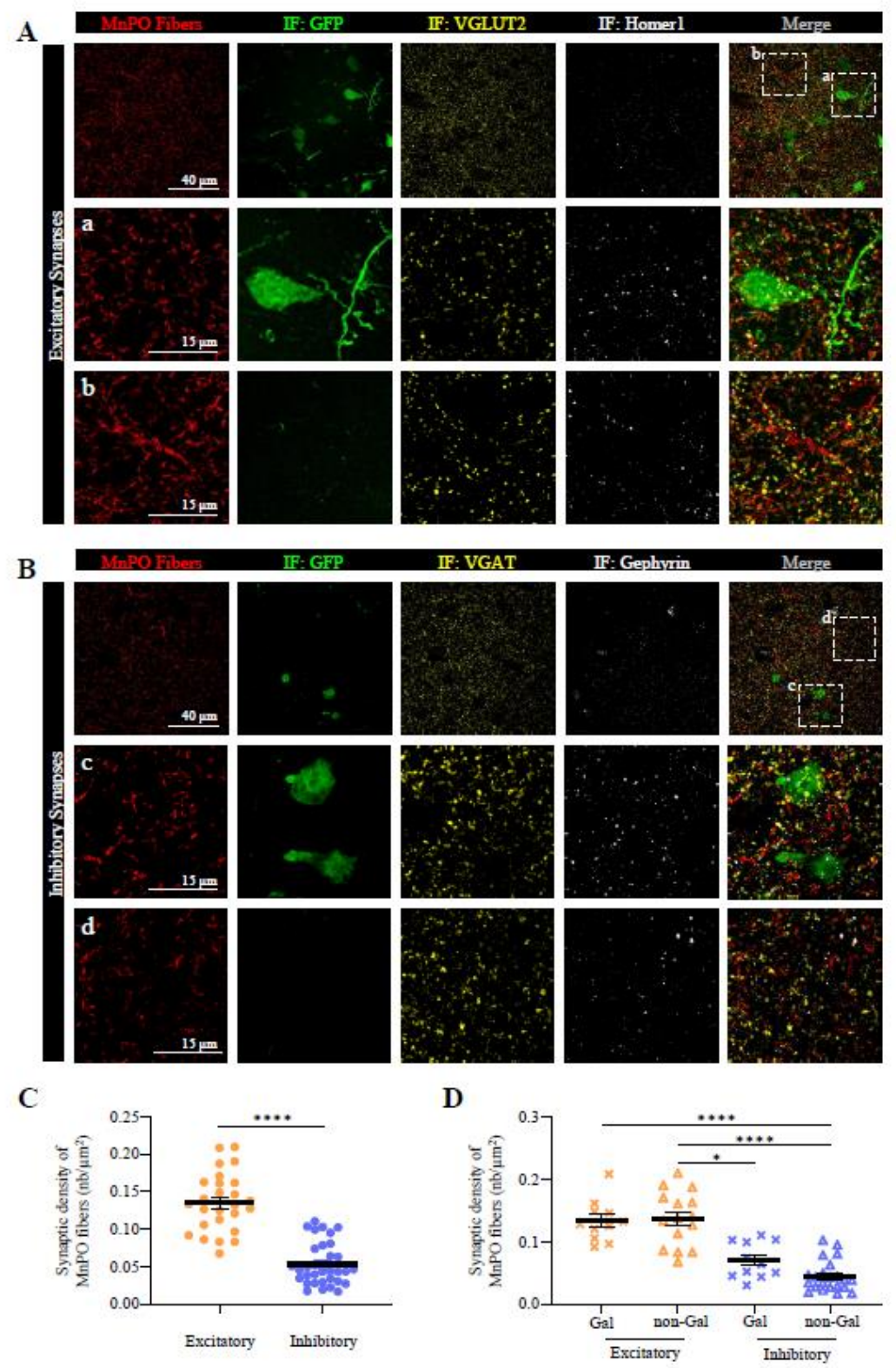

Figure 5. Immunohistological determination of the inhibitory and excitatory synaptic densities on MnPO fibers in the VLPO. A, Representative confocal images of excitatory synapses in the VLPO. MnPO fibers are visualized by their biocytin staining (red). Gal-GFP neurons increased staining was performed using GFP detection (green), presynaptic elements were immunostained for VGluT2 (yellow) and the postsynaptic elements for Homer1 (white). B, Same as in A, but for inhibitory synapses in the VLPO. Presynaptic elements were immunostained for VGat (yellow) and the postsynaptic elements for Gepherin (white). $\mathbf{C}$ and C, Plots of excitatory and inhibitory synapse quantifications performed by MnPO fibers in the VLPO. ****P $<0.0001$, Mann-Whitney test. D, Plots of excitatory and inhibitory synapse quantifications performed by MnPO fibers on non Gal-GFP and Gal-GFP neurons in the VLPO. $* \mathrm{P}<0.05, * * * * \mathrm{P}<0.0001$, Kruskal-Wallis test. 


\section{Propagation of the electrical signal along the axonal track from the MnPO to the VLPO}

We next tested whether the pathway from the MnPO to the VLPO was functional. To do so, we prepared $400 \mu \mathrm{m}$-thick slices from P30 wild type mice, using an angle of $70^{\circ}$ in order to preserve the entire track integrity. The slice of interest, containing both the MnPO and the VLPO was then placed on a multielectrode array (MEA) containing 100 electrodes, each spaced by $100 \mu \mathrm{m}$ (Fig. 6B). As the MEA area could not cover the entire axonal track from the MnPO to the VLPO, we placed the grid on the VLPO and performed stimulation with an electrode that was located the nearest possible to the MnPO (Fig. 6B). We then performed a single stimulation and recorded the signal propagation. The amplitudes of the responses were then measured and color-coded as represented in Figure 6A. We established that signal propagation occurs prominently along the MnPO-VLPO track. We indeed compared the response amplitudes evoked in the VLPO and in another brain region as a control (indicated by gray squares), outside the track and closer to the stimulating electrode that favors passive signal propagation. As illustrated in figure 6C, mean response recorded in the VLPO from 8 slices was higher by $35 \%$ compared to the control region $(25.23 \pm 0.7$ vs $18.61 \pm 0.7, \mathrm{P}<0.001$, Wilcoxon test $)$.

A

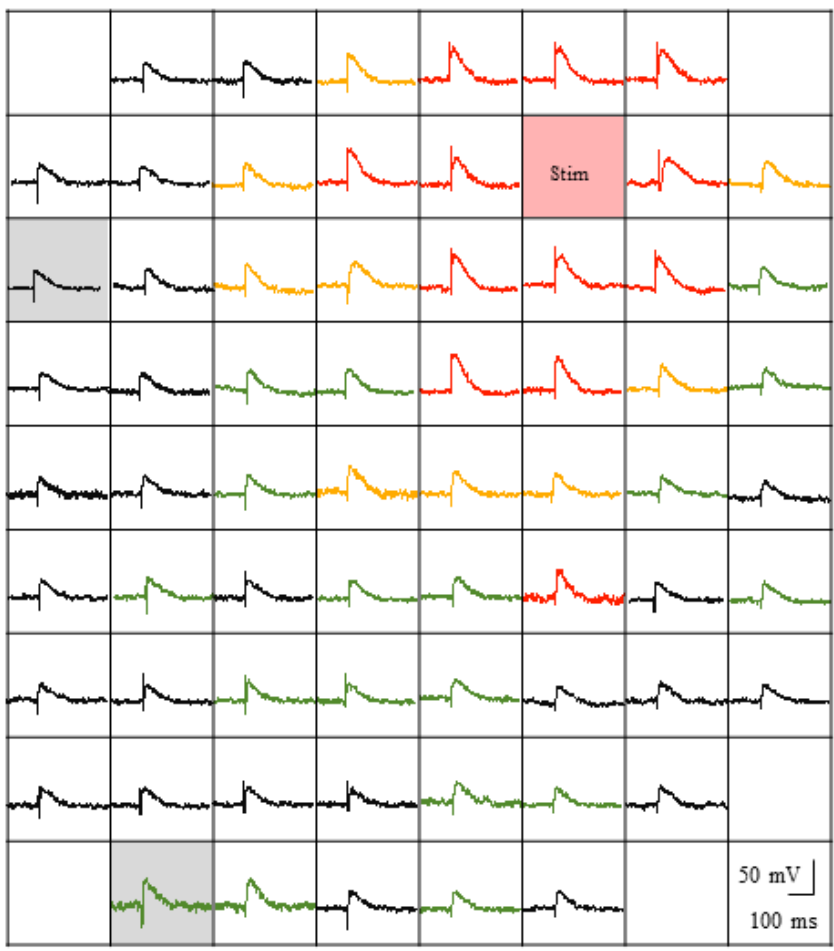

$<25 \mathrm{mV}$

$25-31 \mathrm{mV}$ $31-38 \mathrm{mV}$ $>38 \mathrm{mV}$

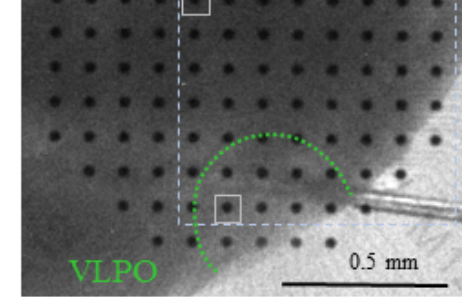

$\mathrm{C}$

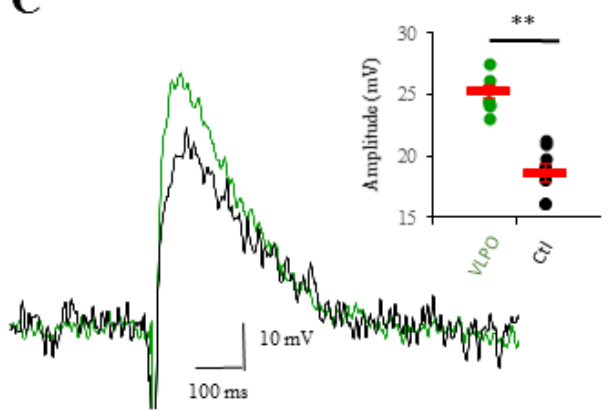


Figure 6. Propagation of electrical signals along the axonal pathway from the MnPO to the VLPO. A, Illustration of a typical extracellular recording with MEA in a MnPO-VLPO slice cut with a $70^{\circ}$ angle. The stimulating electrode is highlighted by a red square. Amplitudes of the responses are color coded. Note that the response propagates throughout the slice from the stimulating electrode to the VLPO. B, Picture of the brain slice (left hemisphere) upon the MEA grid (120 x 120 arrays; $100 \mu \mathrm{m}$ inter-electrode distance), indicating locations of the VLPO (green dash line), of the stimulating electrode (red circle), of a control trace outside the MnPO-VLPO pathway (top left gray circle) and of a response into the VLPO (bottom left circle). Both recorded regions indicated by a gray circle are highlighted in gray in panel (A). C, Superimposed averaged responses recorded in the VLPO $(n=8$, in green) and in a control area ( $n=8$, in black) with the electrodes highlighted in gray in panel (A) or indicated by a gray circle in panel (B). Insert: dots plot of individual responses amplitude recorded in the VLPO $(\mathrm{n}=8$, in green) or in the control region $(\mathrm{n}=8$, in black). Mean values \pm SEM are represented by the red bar, $\mathrm{P}<0.001$ Wilcoxon test.

\section{Functionality of the MnPO-VLPO Pathway}

To further investigate whether an electrical stimulation into the MnPO could propagate until the VLPO and activate local networks, we recorded the field potential in the VLPO evoked by the stimulation of the MnPO, on $70^{\circ} \mathrm{MnPO}-\mathrm{VLPO}$ slices of $400 \mu \mathrm{m}$ thickness (Fig. 7A). We assessed the input-output curves of field postsynaptic potential (fPSP) responses. Fibers from the MnPO were stimulated by positioning the stimulating electrode near the MnPO and the recording electrode was positioned in the VLPO as represented in the figure 7A. Extracellular field potentials revealed excitability variations along the stimulation intensity (Fig. 7B and E) that is proportional to the fiber volley amplitude (Fig. 7C). As shown in the figure 7D and F, field responses were significantly reduced by $54.7 \%$ when $\mathrm{GABA}_{\mathrm{A}}$ receptors were inhibited (picrotoxin $100 \mu \mathrm{M} ; \mathrm{n}=7, \mathrm{P}=0.0156$, Wilcoxon test), and were further reduced, although not significantly, by $35.4 \%$ when blocking NMDA receptors $(\mathrm{CPP}, 100 \mu \mathrm{M})$ and AMPA receptors (NBQX $100 \mu \mathrm{M} ; \mathrm{F}=0.58$, Friedman test; Fig. 7F). However, the field potential response in the presence of these blockers of inhibitory and excitatory synaptic transmission was significantly 
different from the control $(\mathrm{F}=0.0026$, Friedman test $)$. Finally, we demonstrated that these field responses indeed resulted from neuronal activity because they were inhibited when action potential discharge was inhibited using TTX $(1 \mu \mathrm{M} ; \mathrm{n}=7 ; \mathrm{F}=0.0056$, Friedman test; Fig. 7F).

A

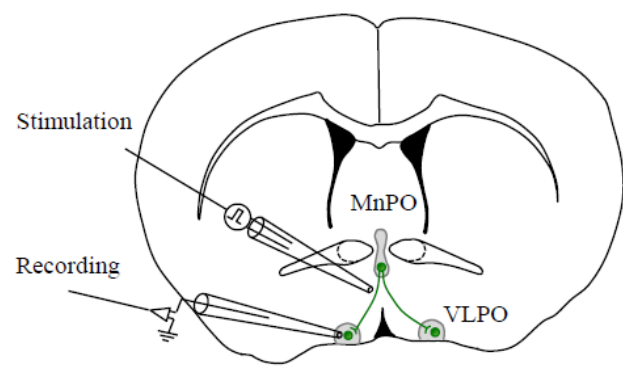

D



B

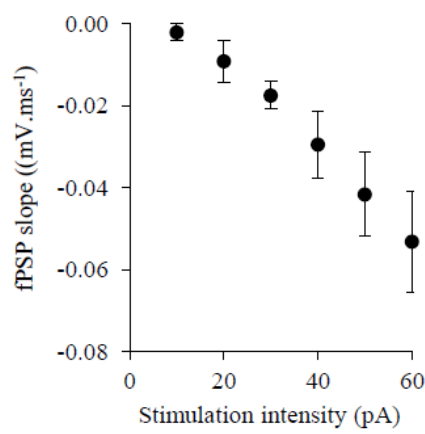

C
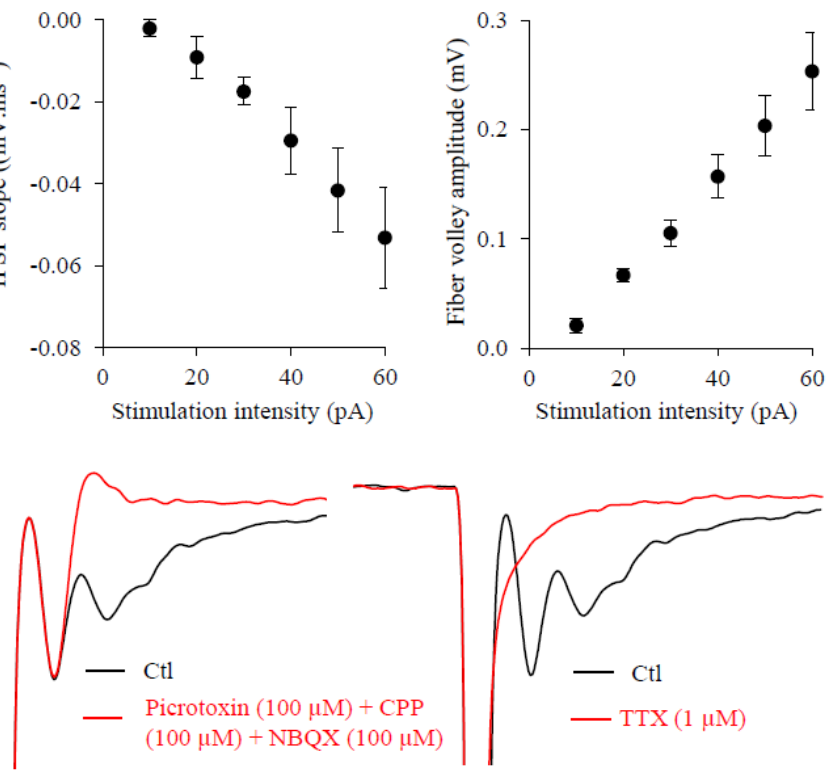

F

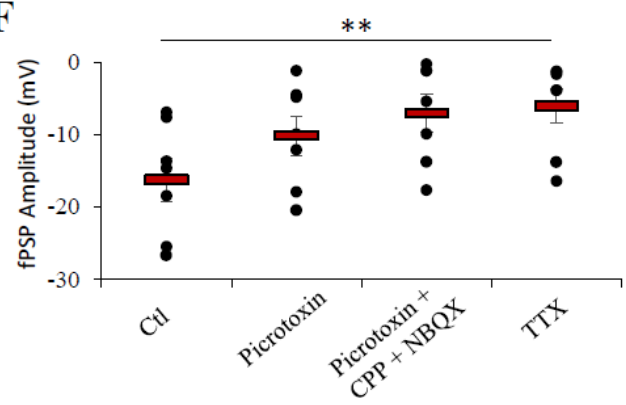

Figure 7. Fiber volley propagates from the MnPO to the VLPO. A, Schematic drawing of an acute MnPO-VLPO slice. MnPO axonal projections toward the VLPO are represented in green and were stimulated as indicated by a glass electrode. Field recordings were performed in the VLPO by a recording electrode. B, Input/output curve of the field PSP slope compared to the stimulation intensity. Individual plots represent the mean \pm SEM ( $n=8$ slices). $C$, Input/output curve of the fiber volley amplitude compared to the stimulation intensity. Individual plots represent the mean \pm SEM ( $n=8$ slices). $D$, Superimposed response evoked at $50 \mathrm{pA}$ in control condition (black trace, all panel) or in the presence of picrotoxin (red trace, left panel), in the presence of picrotoxin plus CPP and NBQX (middle panel) or in the presence 
of TTX (red trace, right panel). E, Superimposed example of volley responses recorded at different stimulation intensity (10, 30, 40, 60 and $70 \mathrm{pA}$; top panel). F, Dot plots of individual recordings (black circles) of fPSP amplitude evoked by a stimulation intensity of $50 \mathrm{pA}$, in control condition $(\mathrm{Ctl})$, or consecutively under picrotoxin $(100 \mu \mathrm{M})$, under picrotoxin plus CPP $(100 \mu \mathrm{M})$ and NBQX $(100 \mu \mathrm{M})$, and under TTX $(1 \mu \mathrm{M} ; \mathrm{n}=7, \mathrm{P}=0.0012$, Friedman test $)$.

\section{Discussion}

We here describe for the first time a MnPO-VLPO slice preparation in which the integrity of the axonal track is preserved. By combining viral infection, biocytin labeling, MEA and field recordings, we here established for the first time that an oblique coronal slice of $70^{\circ}$ preserve the anatomical and functional connectivity between the MnPO and the VLPO.

In this study, we used two different techniques to develop a preparation with intact connectivity between the MnPO and the VLPO. First, we performed in vivo viral infection of MnPO neurons to label efferent axons and used iDISCO method to immunolabel and image the axonal track. After the reconstruction of the MnPO-VLPO pathway, we determined the best angle and thickness for slicing using an algorithm. Then, in a second set of experiments we used crystal depositions on acute slices to investigate whether and how the axonal track from the MnPO to the VLPO was preserved. The aim of this experiment was to ensure that the observed labeled axons were exclusively present in the thickness of the slice, without alternative trajectory. Crystal depositions were therefore performed in vitro on acute and thin slices of $250 \mu \mathrm{m}$ to ensure the specificity of the trajectory.

Using both techniques, we demonstrate that $70^{\circ}$ slice preparation preserved the MnPO-VLPO pathway on all slices containing the VLPO. Using Gal-GFP mice, we located the VLPO by the fluorescence of Gal-GFP neurons that forms a cluster into the VLPO and allows its precise localization. In contrast, we observed in coronal slices that only the rostral part of the VLPO is 
targeted by biocytin labeled fibers from the MnPO. Moreover, we established that the MnPO fiber track was denser on $70^{\circ}$ angular brain sections than on coronal slices. Far more fibers reached the VLPO in these $70^{\circ}$ slice preparations compared to what was observed in coronal sections. Altogether, these experiments show that the oblique slice of $70^{\circ}$ can preserve the connectivity between the MnPO and the VLPO. Neurons from the MnPO also target other brain regions, i.e. the dorsal and median raphe, the locus cœruleus, the tuberomammillary nucleus (TMN), the basal forebrain (BF) and the orexin neurons (Sherin et al., 1998; Uschakov et al., 2007). However, these other pathways were not visible in our slice preparation as they follow a different trajectory.

Functional studies, using MEA or field recording, were subsequently carried out on $400 \mu \mathrm{m}$ thick slices to favor the integrity of the pathway, as it is classically used for these experiments (MacLean et al. 2006; Pannasch et al. 2014; Chever et al. 2016). Using MEA experiments, we established that evoked responses generated near the MnPO preferentially propagate towards the VLPO, following the track previously highlighted by biocytin labeling.

The functionality of the MnPO-VLPO pathway was then assessed by field potential recordings. Although we cannot exclude the possibility that other passing fibers originating from other brain regions could have been recruited by our stimulation, we demonstrated that this electrical stimulation of the axonal track from the MnPO induced a field response in the VLPO. Interestingly, we found that this field response was strongly reduced by $\mathrm{GABA}_{\mathrm{A}}$ receptor antagonist, whereas in the presence of glutamatergic receptor antagonists in addition to $\mathrm{GABA}_{\mathrm{A}}$ receptors antagonist, a non-significant reduction of $34 \%$ was observed, suggesting that GABAergic neurons are mainly involved in field responses. These results are consistent with previous reports indicating that MnPO neurons (Gong et al. 2004), as well as sleep-promoting neurons from the VLPO (Sherin et al. 1996; Sherin et al. 1998) and local interneurons contain GABA (Williams et al. 2014). However, neurons in the MnPO also contain glutamatergic 
neurons. GABAergic and glutamatergic neurons of the $\mathrm{MnPO}$ were reported to play opposite functions (Abbott et al. 2016). Therefore, we investigated the distribution and repartition of excitatory and inhibitory synapses performed by MnPO fibers into the VLPO. We found that afferent MnPO fibers preferentially make inhibitory synaptic contacts on Gal-GFP neurons compared to non-Gal-GFP. However, we observed a higher density of glutamatergic synapses made by MnPO compared to inhibitory synapses, globally and in Gal-GFP neurons vs. non GalGFP neurons, suggesting a complex regulation of neuronal activity within the VLPO.

As extracellular field potentials reflect the collective membrane depolarization, we hypothesize that the GABAergic input from the MnPO could disinhibit local neurons to generate a field response. Indeed, responses recorded in the VLPO are small, compared to recordings in the hippocampus, where the majority of the neuronal network is constituted by excitatory neurons (Ross et al. 2000). Thus, GABAergic afferent inputs from the MnPO could disinhibit VLPO network to turn off arousal-promoting brain regions (Saper et al. 2010; Scammell et al. 2017). Alternatively, glutamatergic inputs from the MnPO could also indirectly or directly stimulate sleep-promoting neurons to favor NREM sleep. A better characterization of the neuronal circuitry within the VLPO would greatly benefit to the understanding of sleep regulation.

Single-unit recordings and c-Fos expression revealed that the MnPO and the VLPO contain NREM sleep active neurons (Sherin et al. 1996; Szymusiak et al. 1998; Suntsova et al. 2002; Takahashi et al. 2009; Alam et al. 2014). Although it has been suggested that MnPO neurons might be implicated in sleep pressure whereas VLPO neurons would rather be involved in sleep onset and maintenance, these nuclei contain different neuronal subtypes with distinct physiological roles, i.e. regulation of vigilance states, osmolarity or body temperature (McKinley et al. 2015; Abbott and Saper 2017; Zhang et al. 2017; Kroeger et al. 2018). Indeed, galaninergic neurons from the VLPO were already shown to project toward brain regions that regulate both sleep-wake behavior and body temperature. Moreover, their chemogenetic 
activation and their low frequency stimulation using optogenetic tools were recently shown to promote both sleep and heat loss (Kroeger et al. 2018). Neuronal circuitries within the preoptic area are complex and regulate numerous fundamental functions associated with sleep regulation. However, the underlying circuit mechanism remains poorly understood.

Therefore, the slice preparation preserving the connection between the MnPO and the VLPO that we have here characterized for the first time, could be used in future studies to explore the physiological role of MnPO inputs on VLPO local interneurons and sleep-promoting neurons. It would be interesting to determine which type of neurons receive direct inputs from the MnPO, and whether specific spatiotemporal patterns of activity in the MnPO pathway can generate neuronal responses in the VLPO.

\section{Acknowledgements}

This work was supported by the Centre National de la Recherche Scientifique (CNRS), the Institut National de la Santé et de la Recherche Médicale (Inserm) and Collège de France. We thank all the members of the animal house facility and imaging platform from Collège de France, including Philippe Mailly in particular. We gratefully acknowledge Yves Dupraz for the design of the brain cutting angles and IT people including Aurélien Agneray, in particular, for excellent and prompt technical assistance.

\section{Compliance with ethical standards}

Conflict of interest The authors have no conflicts of interest to declare. 
Ethical approval All animal procedures were carried out according to the guidelines of European Community Council Directives of January first 2013 (2010/63/EU) and followed our local guidelines for the ethical treatment of animal care (Center for Interdisciplinary Research in Biology in College de France (France)). The number of animals in our study was kept to the necessary minimum. 


\section{References}

Abbott SBG, Machado NLS, Geerling JC, Saper CB (2016) Reciprocal Control of Drinking Behavior by Median Preoptic Neurons in Mice. J Neurosci 36:8228-8237. doi: 10.1523/jneurosci.1244-16.2016

Abbott SBG, Saper CB (2017) Median preoptic glutamatergic neurons promote thermoregulatory heat loss and water consumption in mice. J Physiol 595:6569-6583. doi: $10.1113 / \mathrm{JP} 274667$

Alam MA, Kumar S, McGinty D, et al (2014) Neuronal activity in the preoptic hypothalamus during sleep deprivation and recovery sleep. J Neurophysiol 111:287-99. doi: 10.1152/jn.00504.2013

Anaclet C, Ferrari L, Arrigoni E, et al (2014) The GABAergic parafacial zone is a medullary slow wave sleep-promoting center. Nat Neurosci 17:1217-24. doi: 10.1038/nn.3789

Chever O, Dossi E, Pannasch U, et al (2016) Astroglial networks promote neuronal coordination. Sci Signal 9:1-9. doi: 10.1126/scisignal.aad3066

Chou TC, Bjorkum AA, Gaus SE, et al (2002) Afferents to the ventrolateral preoptic nucleus. J Neurosci 22:977-990.

Economo C V. (1930) Sleep As a Problem of Localization. J Nerv Ment Dis 71:249-259. doi: 10.1097/00005053-193003000-00001

Franklin KBJ, Paxinos G (2007) The mouse brain in stereotaxic coordinates, 3rd edn. Academic Press, San Diego.

Gaus SE, Strecker RE, Tate BA, et al (2002) Ventrolateral preoptic nucleus contains sleepactive, galaninergic neurons in multiple mammalian species. Neuroscience 115:285-294.

Gong H, McGinty D, Guzman-Marin R, et al (2004) Activation of c-fos in GABAergic neurones in the preoptic area during sleep and in response to sleep deprivation. J Physiol 556:935-46. 
Gong S, Zheng C, Doughty ML, et al (2003) A gene expression atlas of the central nervous system based on bacterial artificial chromosomes. Nature 425:917-25. doi: 10.1038/nature02033

Gvilia I, Xu F, McGinty D, Szymusiak R (2006) Homeostatic regulation of sleep: a role for preoptic area neurons. J Neurosci 26:9426-33. doi: 10.1523/JNEUROSCI.2012-06.2006

Kroeger D, Absi G, Gagliardi C, et al (2018) Galanin neurons in the ventrolateral preoptic area promote sleep and heat loss in mice. Nat Commun. doi: 10.1038/s41467-018-06590-7

MacLean JN, Fenstermaker V, Watson BO, Yuste R (2006) A visual thalamocortical slice. Nat Methods 3:129-134. doi: 10.1038/nmeth849

McKinley MJ, Yao ST, Uschakov A, et al (2015) The median preoptic nucleus: front and centre for the regulation of body fluid, sodium, temperature, sleep and cardiovascular homeostasis. Acta Physiol (Oxf) 214:8-32. doi: 10.1111/apha.12487

Pannasch U, Freche D, Dallérac G, et al (2014) Connexin 30 sets synaptic strength by controlling astroglial synapse invasion. Nat Neurosci 17:549-58. doi: 10.1038/nn.3662

Renier N, Wu Z, Simon DJ, et al (2014) IDISCO: A simple, rapid method to immunolabel large tissue samples for volume imaging. Cell 159:896-910. doi: 10.1016/j.cell.2014.10.010

Ross FM, Cassidy J, Wilson M, Davies SN (2000) Developmental regulation of hippocampal excitatory synaptic transmission by metabotropic glutamate receptors. Br J Pharmacol 131:453-464. doi: 10.1038/sj.bjp.0703610

Sakai K, Crochet S (2001) Differentiation of presumed serotonergic dorsal raphe neurons in relation to behavior and wake-sleep states. Neuroscience 104:1141-1155.

Saper CB, Fuller PM, Pedersen NP, et al (2010) Sleep state switching. Neuron 68:1023-1042.

Saper CB, Scammell TE, Lu J (2005) Hypothalamic regulation of sleep and circadian rhythms. Nature 437:1257-1263.

Scammell TE, Arrigoni E, Lipton JO (2017) Neural Circuitry of Wakefulness and Sleep. 
Neuron 93:747-765. doi: 10.1016/j.neuron.2017.01.014

Scharbarg E, Daenens M, Lemaître F, et al (2016) Astrocyte-derived adenosine is central to the hypnogenic effect of glucose. Sci Rep 6:19107.

Sherin JE, Elmquist JK, Torrealba F, Saper CB (1998) Innervation of histaminergic tuberomammillary neurons by GABAergic and galaninergic neurons in the ventrolateral preoptic nucleus of the rat. J Neurosci 18:4705-4721.

Sherin JE, Shiromani PJ, McCarley RW, Saper CB (1996) Activation of ventrolateral preoptic neurons during sleep. Science 271:216-9.

Suntsova N, Szymusiak R, Alam MN, et al (2002) Sleep-waking discharge patterns of median preoptic nucleus neurons in rats. J Physiol 543:665-677. doi: 10.1113/jphysiol.2002.023085

Szymusiak R, Alam N, Steininger TL, McGinty D (1998) Sleep-waking discharge patterns of ventrolateral preoptic/anterior hypothalamic neurons in rats. Brain Res 803:178-88.

Takahashi K, Lin J-S, Sakai K (2009) Characterization and mapping of sleep-waking specific neurons in the basal forebrain and preoptic hypothalamus in mice. Neuroscience 161:26992. doi: 10.1016/j.neuroscience.2009.02.075

Thompson RH, Swanson LW (2003) Structural characterization of a hypothalamic visceromotor pattern generator network. Brain Res Brain Res Rev 2003;41:153-202.

Uschakov A, Gong H, McGinty D, Szymusiak R (2007) Efferent projections from the median preoptic nucleus to sleep- and arousal-regulatory nuclei in the rat brain. Neuroscience 150:104-20.

Uschakov A, Gong H, McGinty D, Szymusiak R (2006) Sleep-active neurons in the preoptic area project to the hypothalamic paraventricular nucleus and perifornical lateral hypothalamus. Eur J Neurosci 23:3284-3296.

Williams RH, Chee MJS, Kroeger D, et al (2014) Optogenetic-mediated release of histamine 
reveals distal and autoregulatory mechanisms for controlling arousal. J Neurosci 34:60239. doi: 10.1523/JNEUROSCI.4838-13.2014

Zhang W, Zhou Q, Chen W, et al (2017) A hypothalamic circuit that controls body temperature. Proc Natl Acad Sci 114:E1755-E1755. doi: 10.1073/pnas.1701881114 